OPEN ACCESS

Edited by:

Guan Zhu,

Texas A\&M University, USA

Reviewed by:

Carol Geralyn Chitko-McKown, Agricultural Research Service (USDA),

USA

Henry M. Staines,

St George's, University of London, UK

*Correspondence:

Junlong Liu

liujunlong@caas.cn

Specialty section:

This article was submitted to

Infectious Diseases,

a section of the journal

Frontiers in Microbiology

Received: 07 December 2016 Accepted: 28 February 2017

Published: 14 March 2017

Citation:

Liu J, Guan G, Li Y, Liu A, Luo J and Yin $H$ (2017) A Molecular Survey

of Babesia Species and Detection of a New Babesia Species by DNA Related to $B$. venatorum from White Yaks in Tianzhu, China.

Front. Microbiol. 8:419.

doi: 10.3389/fmicb.2017.00419

\section{A Molecular Survey of Babesia Species and Detection of a New Babesia Species by DNA Related to $B$. venatorum from White Yaks in Tianzhu, China}

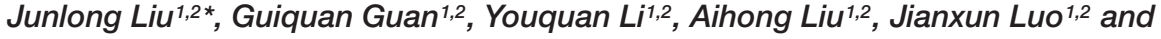 \\ Hong Yin ${ }^{1,2}$
}

${ }^{1}$ State Key Laboratory of Veterinary Etiological Biology, Key Laboratory of Veterinary Parasitology of Gansu Province, Lanzhou Veterinary Research Institute, Chinese Academy of Agricultural Sciences, Lanzhou, China, ${ }^{2}$ Jiangsu Co-innovation Center for Prevention and Control of Important Animal Infectious Diseases and Zoonoses, Yangzhou, China

Bovine babesiosis is a tick-transmitted disease caused by different species of Babesia. The white yak is a unique yak breed that lives only in Tianzhu in the Tibetan Autonomous County, Gansu Province, in northwestern China. Previous research using the ELISA method has confirmed that the white yak could become infected with $B$. bigemina. The objective of this study was the molecular detection and identification of Babesia species in white yaks. A total of 409 white yak blood samples were collected from 11 areas of the Tianzhu Tibetan Autonomous County in Northwest China from April to August, 2015. The V4 hypervariable region of Babesia 18S rRNA was amplified from extracted genomic DNA using nested PCR and sequenced. The nearly full-length sequence of 18S rRNA including the V4 region from the newly discovered Babesia was amplified and sequenced with Sanger method. PCR detection and sequencing indicated that 4/409 samples were positive for B. bigemina, 3/409 samples were positive for $B$. bovis, and 5/409 samples were positive for $B$. ovata. Additionally, a new Babesia species was found in 4/409 white yaks. A unique sequence of 1,627 bp was obtained from two of the four samples. The sequence was similar to Babesia species Akita (98.5\%) found in Ixodes ovatus and B. venatorum (98\%) and shared a $98 \%$ identity with $B$. divergens and a $98.1 \%$ identity with $B$. odocoilei. This study provides new data about Babesia infections in white yaks in northwestern China, and a new Babesia species similar to $B$. venatorum was identified in white yaks for the first time.

Keywords: white yak, Babesia, Babesia venatorum, 18S rRNA, Northwest China

\section{INTRODUCTION}

Bovine babesiosis is a tick-borne disease caused by the intra-erythrocytic apicomplexan protozoan parasites of the different species of Babesia. B. bovis and B. bigemina are the most common bovine babesiosis pathogens and have an important economic and veterinary impact in ruminants (Kim et al., 2008). These and other Babesia species, including B. ovata, B. major, B. orientalis, and Babesia sp. Kashi, have been confirmed to exist in China (Yin et al., 1997; Luo et al., 2002; Liu et al., 2008). 
In addition, some Babesia species can infect both humans and animals, one of which is $B$. divergens, the primary agent of human babesiosis in Europe (Malandrin et al., 2010). In China, two patients from Shandong Province were confirmed to be infected with $B$. divergens (Qi et al., 2011), but B. divergens infections in cattle have not been reported in China until now.

The white yak (Bos grunniens) is a special yak breed that mainly lives in Tianzhu in the Tibetan Autonomous County (TTAC), Gansu Province, in northwestern China (Qin et al., 2014). Because TTAC is part of the Qinghai-Tibet Plateau where the weather is dry and cold, white yaks are important to the local residents for things such as meat and milk production. White yaks are considered semi-wild animals in China and thus have more random contact with ticks, which could transmit Babesia. $B$. bigemina is a parasite that can infect cattle and buffalo and was recently confirmed to infect black yaks and white yaks (He et al., 2012; Schnittger et al., 2012; Saravanan et al., 2013; Qin et al., 2015). Additionally, some black yak blood samples from Gannan in the Tibetan Autonomous County were confirmed to be infected with B. bovis by PCR (Niu et al., 2015). However, no systematic data concerning Babesia infections in white yaks have been reported until now. In this study, blood samples from white yaks were collected from 11 areas of the TTAC to determine which species of Babesia might have infected white yaks.

\section{MATERIALS AND METHODS}

\section{Blood Samples}

A total of 409 blood samples were randomly collected from white yaks from 11 areas of the TTAC (Table 1). Blood samples from the jugular vein were collected in tubes containing EDTA and then stored at $4^{\circ} \mathrm{C}$ until DNA extraction. The procedure of the samples collection was similar as described in the paper for detection of Babesia and Theileria (Lempereur et al., 2017).

\section{DNA Extraction}

DNA was extracted from a $300-\mu \mathrm{L}$ aliquot of each blood sample with a Genomic DNA Purification Kit by following

TABLE 1 | The number of positive blood samples for Babesia spp. infection from white yaks from Tianzhu Tibetan Autonomous County, Gansu province.

\begin{tabular}{lccccc}
\hline Location & Total no. & B. bigemina & B. bovis & B. ovata & B. sp \\
\hline Saisishi & 40 & 0 & 1 & 0 & 0 \\
Tiantang & 53 & 3 & 2 & 1 & 1 \\
Maozang & 59 & 0 & 0 & 2 & 3 \\
Dongdatan & 41 & 1 & 0 & 2 & 0 \\
Anyuan & 20 & 0 & 0 & 0 & 0 \\
Xidatan & 25 & 0 & 0 & 0 & 0 \\
Shimen & 30 & 0 & 0 & 0 & 0 \\
Zhuaxixiulong & 31 & 0 & 0 & 0 & 0 \\
Duoshi & 30 & 0 & 0 & 0 & 0 \\
Tanshancen & 30 & 0 & 0 & 0 & 0 \\
Danma & 50 & 0 & 0 & 0 & 0 \\
Total number & 409 & 4 & 3 & 5 & 4
\end{tabular}

the manufacturer's instructions (Qiagen, Hilden, Germany). The extracted DNA was eluted in $100 \mu \mathrm{L}$ of elution buffer, and the concentration was determined with a NanoDrop 2000 spectrophotometer (NanoDrop Technologies, Wilmington, DE, USA). The DNA was stored at $-20^{\circ} \mathrm{C}$ until further analysis.

\section{PCR Amplification of the V4 Region of the 18S rRNA Gene}

Nested PCR was used to amplify the V4 hypervariable region of the 18S rRNA of Babesia using previously reported universal primers (Gubbels et al., 1999; Centeno-Lima et al., 2003; Zanet et al., 2014; Liu et al., 2016). The first PCR reaction was conducted with the primers RLB-F2 (5'-GACACAGGGAGGTAGTGACAAG-3') and RLB-R2 $\left(5^{\prime}\right.$-CTAAGAATTTCACCTCTGACAGT- $\left.3^{\prime}\right)$ in a $25 \mu \mathrm{L}$ total volume containing $12.5 \mu \mathrm{L}$ Premix Taq DNA Polymerase (TaKaRa, Dalian, China), $1 \mu \mathrm{M}$ of each primer and $1 \mu \mathrm{L}$ of genomic DNA. The PCR reaction was started with a one-step initial denaturation at $95^{\circ} \mathrm{C}$ for $3 \mathrm{~min}$, which was followed by 35 cycles of denaturation at $95^{\circ} \mathrm{C}$ for $1 \mathrm{~min}$, $52^{\circ} \mathrm{C}$ for $50 \mathrm{~s}$ and extension at $72^{\circ} \mathrm{C}$ for $1 \mathrm{~min}$, with a final extension at $72^{\circ} \mathrm{C}$ for $5 \mathrm{~min}$. The primers RLB-FINT $\left(5^{\prime}-\right.$ GACAAGAAATAACAATACRGGGC-3') and RLB-R2 were, respectively, used as the forward and reverse primers in a second PCR. The reaction mixture had the same composition as in the first PCR, except that the template was replaced by $1 \mu \mathrm{L}$ of the first PCR product. The reaction cycling comprised an initial denaturation step of $95^{\circ} \mathrm{C}$ for $3 \mathrm{~min}$, followed by 35 cycles of denaturation $\left(95^{\circ} \mathrm{C}\right.$ for $\left.30 \mathrm{~s}\right)$, primer annealing $\left(50^{\circ} \mathrm{C}\right.$ for $\left.30 \mathrm{~s}\right)$ and extension $\left(72^{\circ} \mathrm{C}\right.$ for $\left.30 \mathrm{~s}\right)$. A final extension was performed at $72^{\circ} \mathrm{C}$ for $5 \mathrm{~min}$. Each PCR product was electrophoresed on a $1.5 \%$ agarose gel containing $10 \mu \mathrm{L}$ of gold view dye (SolarBio, Tianjin, China) in Tris-acetate-EDTA (TAE) buffer at $120 \mathrm{~V}$ for $30 \mathrm{~min}$ and visualized under UV light.

The positive PCR products were cloned into the pGEM-T Easy vectors (Promega, USA) and transformed into Escherichia coli JM109 (TaKaRa, China). At least five positive clones were sequenced using an ABI Prism Terminator Cycle Sequencing kit and carried out on an Applied Biosystem 3730 DNA Analyzer (Sangon Biotech, Shanghai, China) to obtain consensus sequences.

\section{PCR Amplification of the Long Fragment of $18 S$ rRNA}

A long sequence of $18 \mathrm{~S}$ rRNA which containing the V4 region was amplified with nested PCR, to clarify the classification of the newly discovered Babesia. The first round PCR was amplified using the primer set PiroF $\left(5^{\prime}\right.$-GCCAGTAGTC ATATGCTTGTGTTA-3 $\left.{ }^{\prime}\right)$ and Piro6R (5' ${ }^{\prime}$-CTCCTTCCTYTAAG TGATAAGGTTCAC-3'). Another pair of primers, PirolF (5'CCATGCAGTTCTWAGTAYAARCTTTTA-3') and Piro5.5R (5'-CCTYTAAGTGATAAGGTTCACAAAACTT-3') were used in the second PCR (Kawabuchi et al., 2005). The composition of the PCR reactions was the same described as above. The PCR reactions were initiated with one step of $95^{\circ} \mathrm{C}$ for $3 \mathrm{~min}$, followed by 35 cycles of denaturation $\left(94^{\circ} \mathrm{C}\right.$ for $\left.1 \mathrm{~min}\right)$, primer 
annealing $\left(59^{\circ} \mathrm{C}\right.$ for $\left.1 \mathrm{~min}\right)$ and extension $\left(72^{\circ} \mathrm{C}\right.$ for $\left.2 \mathrm{~min}\right)$. A final extension was performed at $72^{\circ} \mathrm{C}$ for $10 \mathrm{~min}$. The cloning and sequencing procedure was the same as described above.

\section{Sequence Analysis}

The sequences obtained were aligned with the related Babesia sp. $18 \mathrm{~S}$ rRNA by using the MegAlign component of the DNAStar software program (Version 4.0 DNAStar, Madison, WI, USA). The section containing the cloning vector sequence was manually removed. The final $18 \mathrm{~S}$ rRNA sequences were submitted to the GenBank database.

A phylogenetic analysis of the $18 \mathrm{~S}$ rRNA genes determined in this study with other sequences registered in GenBank was carried out with MEGA 6.0 software. The distance matrices for the aligned sequences were calculated by the Kimura twoparameter method, and the neighbor-joining method was used to generate a phylogenetic tree (Kimura, 1980; Tamura et al., 2013).

\section{Ethical Approval}

The present work was approved by the Animal Ethics Committee of Lanzhou Veterinary Research Institute CAAS (No. LVRIAEC2013-010). The procedures for acquiring the field samples were in accordance with the Animal Ethics Procedures and Guidelines of China.

\section{RESULTS}

Genomic DNA was successfully extracted from the blood samples, and the positive PCR products that targeted the $18 \mathrm{~S}$ rRNA V4 region of the piroplasms were sequenced. The sequences were then phylogenetically analyzed with related Babesia species 18S rRNA gene sequences deposited in GenBank. The analysis of results identified four samples from Tiantang and Dongdatan as B. bigemina (GenBank accession no. KX870088, KX870089, KX870090, and KX870091). The other three samples from Saisishi and Tiantang were grouped with B. bovis (GenBank accession no. KX870092, KX870093 and KX970094). The five samples from Tiantang, Maozang and Dongdatan, were identical with B. ovata (GenBank accession no. KX870095, KX870096, KX870097, KX870098, and KX870099). The four samples from Tiantang and Maozang were closely related to B. venatorum, B. odocoilei, and B. sp Akita that was identified in Ixodes ovatus from Japan (GenBank accession no. KX870100, KX870101, KX870102, and KX870103) (Table 1 and Figure 1). No co-infections were observed in the samples analyzed.

To confirm the classification of the newly discovered Babesia species that was identified based on the V4 region of $18 \mathrm{~S}$ rRNA, a nearly full-length $18 \mathrm{~S}$ rRNA gene sequence of Babesia was amplified from the four samples from Tiantang and Maozang. Only two samples from Maozang produced a fragment of approximately $1700 \mathrm{bp}$. After removal of the vector sequence, an identical sequence which contained 1672 bp (GenBank accession no. KX870104) was obtained from those two samples. The sequence shared a $98.5 \%$ identity with B. sp. Akita, a $98.1 \%$ identity with $B$. odocoilei, and a $98 \%$ identity with $B$. venatorum and $B$. divergens. The phylogenetic analysis showed that the

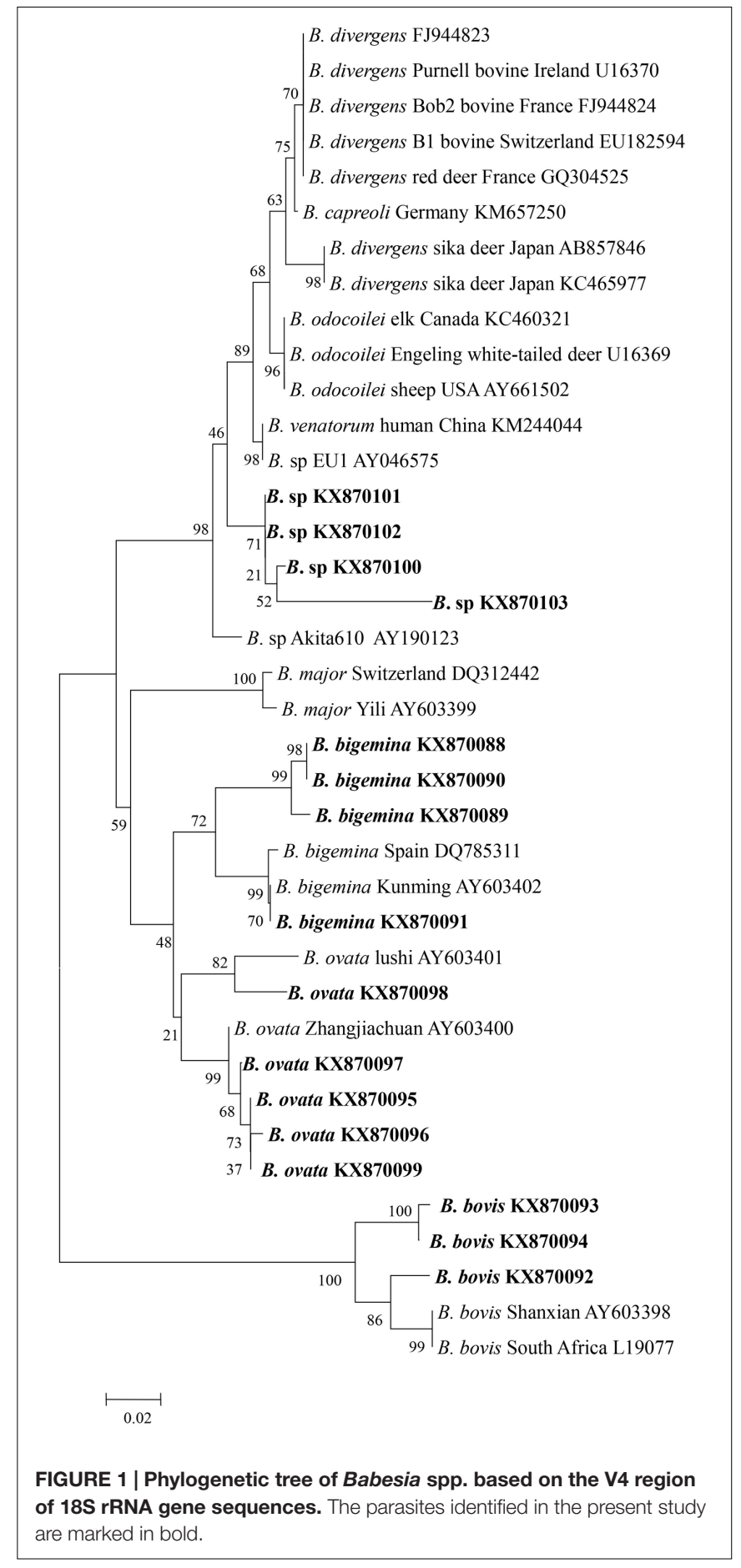

newly discovered Babesia from white yak belonged to the group containing $B$. divergens, $B$. odocoilei, B. venatorum, and B. sp Akita (Figure 2).

\section{DISCUSSION}

In the present study, a total of 409 blood samples from white yaks was collected and tested for Babesia infection. Three species of 


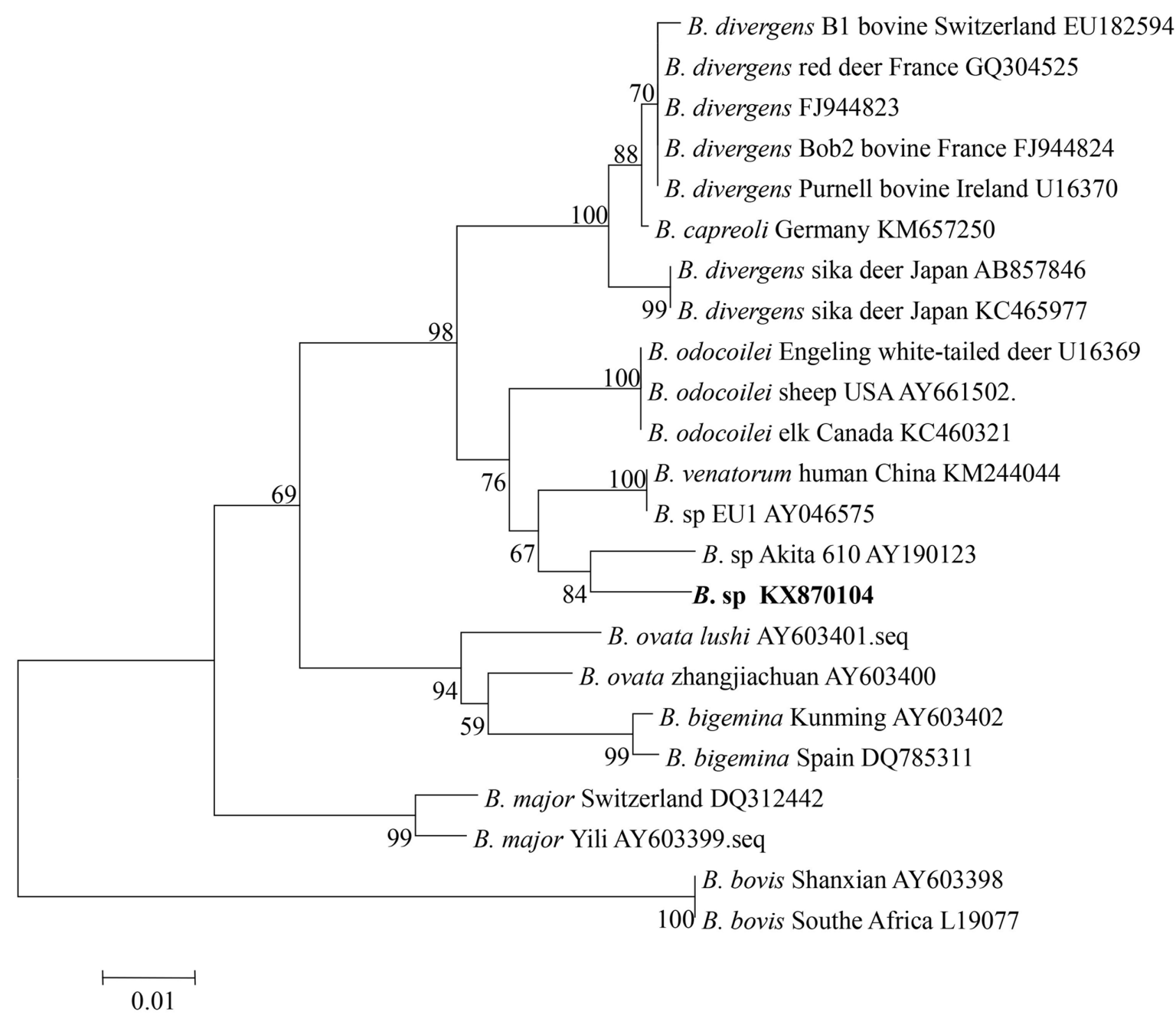

FIGURE 2 | Phylogenetic relationship of various Babesia spp. based on the nucleotide sequences of the 18S rRNA gene. The parasite identified in the present study is marked in bold.

Babesia previously reported in China were identified, including $B$. bovis, B. bigemina, and B. ovata. Because they share the same transmission vector (Rhipicephalus microplus), B. bovis, and $B$. bigemina often co-infect a host. The area with the greatest prevalence of those two parasites is central and southern China, and the primary host is cattle (Liu et al., 2014). In addition to cattle, water buffalo has also been confirmed to be infected with $B$. bovis and B. bigemina by both DNA and serum detection methods (He et al., 2012). A few reports have described the infection of $B$. bigemina in yaks including black and white yaks in China and Nepal by using serological methods (Graves et al., 1975; Qin et al., 2015). Recent studies have confirmed B. bigemina infection in yaks with PCR targeting the 18S rRNA fragment of $B$. bigemina and by further sequencing analysis as well as restriction digestion of a PCR amplified fragment (Saravanan et al., 2013). In the present study, four white yak samples tested positive for B. bigemina genomic DNA. A specific PCR method for amplifying the rhoptry-associated-protein-1 gene was used to detect $B$. bovis in black yaks from the Gannan Tibetan Autonomous Prefecture (GTAP), Gansu Province, which first confirmed the $B$. bovis infection of black yaks. In the present study, three white yaks from the TTAC, which has similar environmental conditions to the GTAP, were identified as infected with B. bovis. This study is the first report of the B. bovis infection of white yaks from the TTAC.

Babesia ovata is mainly transmitted by Haemaphysalis longicornis in China, and it was confirmed to exist in Gansu, Henan, and Sichuan Provinces (Liu et al., 2008). The vertebrate host for B. ovata is cattle, and no other animals have been reported as hosts. $B$. ovata infection was identified in white yaks in this study. Five blood samples from three areas of the TTAC were confirmed as B. ovata positive by sequencing analysis. During blood sample collection, ticks were also collected from both the grass and the yaks, and two species ( $H$. qinghaiensis and $H$. longicornis) were identified, indicating the possibility of B. ovata transmission in the TTAC. 
The phylogenetic analysis grouped Babesia 18S rRNA gene nucleotide sequences from four samples from Tiantang and Maozang with the normal bovine Babesia species but also showed a close relationship with $B$. venatorum and Babesia species Akita 610. These results suggest that the discovered Babesia species might be a new Babesia found in bovines and is the first time that this group of Babesia has been detected in white yaks from Gansu Province. B. venatorum, previously named Babesia sp. EU1, was first reported in two asplenic men from Italy and Austria, and later, a 63-year-old man from Germany was confirmed as infected with B. venatorum (Herwaldt et al., 2003; Häselbarth et al., 2007). This parasite was also identified in reindeer, and I. ricinus was confirmed as the transmission vector (Bonnet et al., 2009; Kik et al., 2011). A recent study has indicated the high prevalence of $B$. venatorum in $I$. persulcatus, suggesting that this tick might an important vector for $B$. venatorum in Mongolia (Karnath et al., 2016). B. venatorum was also found to infect humans in China. In 2012, a 8-year-old child from the Xinjiang Autonomous Region was confirmed as infected with B. venatorum (Sun et al., 2014). Another study described 48 cases, including 32 confirmed cases and 16 probable cases from Heilongjiang Province, of $B$. venatorum infections. In the same study, $B$. venatorum DNA was also detected in $I$. persulcatus (Jiang et al., 2015). In Japan, a Babesia species Akita610 closely related to $B$. venatorum was found in I. ovatus collected from dogs, but Babesia species were not detected in dogs (Inokuma et al., 2003). The tick vectors of $B$. venatorum were not found in the area where the samples were collected for our study. However, in the Gannan Tibetan Autonomous Prefecture, which has a similar climate as the TTAC, a few I. ovatus have been collected (personal communication). Thus, the details of the tick distribution in the TTAC should be determined to clarify the vector for the newly discovered Babesia. Moreover, epidemiological studies of humans infected with this Babesia species are necessary because $B$. venatorum is known as a human pathogen and several cases of $B$. venatorum infection in humans have been reported in European countries and in China.

\section{REFERENCES}

Bonnet, S., Brisseau, N., Hermouet, A., Jouglin, M., and Chauvin, A. (2009). Experimental in vitro transmission of Babesia sp. (EU1) by Ixodes ricinus. Vet. Res. 40:21. doi: 10.1051/vetres/2009004

Centeno-Lima, S., do Rosário, V., Parreira, R., Maia, A. J., Freudenthal, A. M., Nijhof, A. M., et al. (2003). A fatal case of human babesiosis in Portugal: molecular and phylogenetic analysis. Trop. Med. Int. Health 8, 760-764. doi: $10.1046 / j .1365-3156.2003 .01074 . x$

Graves, I. L., Adams, W. H., and Pyakural, S. (1975). Recurring hemolytic anemia, babesiasis, and influenza A viruses in a yak at low altitude in Nepal. Am. J. Vet. Res. 36, 843-845.

Gubbels, J. M., de Vos, A. P., van der Weide, M., Viseras, J., Schouls, L. M., de Vries, E., et al. (1999). Simultaneous detection of bovine Theileria and Babesia species by reverse line blot hybridization. J. Clin. Microbiol. 37, 1782-1789.

Häselbarth, K., Tenter, A. M., Brade, V., Krieger, G., and Hunfeld, K.-P. (2007). First case of human babesiosis in Germany - clinical presentation and molecular characterisation of the pathogen. Int. J. Med. Microbiol. 297, 197-204. doi: 10.1016/j.ijmm.2007.01.002

He, L., Feng, H.-H., Zhang, W.-J., Zhang, Q.-L., Fang, R., Wang, L.-X., et al. (2012). Occurrence of Theileria and Babesia species in water buffalo (Bubalus

\section{CONCLUSION}

In this study, a total of 409 white yak blood samples from the TTAC were used for an epidemiological study of Babesia infection. Three normal bovine Babesia species including B. bigemina, B. bovis, and B. ovata were detected in white yaks, and based on 18S rRNA gene analysis, a new Babesia species closely related to $B$. venatorum and $B$. $s p$ Akita was found in four white yaks. The tick vector $(H$. longicornis) for $B$. ovata was found in this area, but the vectors for $B$. bovis, B. bigemina, and B. venatorum were not found. Thus, more studies must be carried out to study the distribution of tick species in this area and to elucidate the life cycle of $B$. bovis, B. bigemina and the newly discovered Babesia.

\section{AUTHOR CONTRIBUTIONS}

JLL, GG, and AL did the sample collection and the molecular genetic studies. JLL wrote the draft of the manuscript. GG, YL, JXL, and HY corrected the manuscript. All authors read and approved the final manuscript.

\section{ACKNOWLEDGMENTS}

This study was financially supported by the National Key Basic Research Program (973 Program) of China (2015CB150300); NSFC (№31402189, №31372432), the Science Fund for Youth of Gansu Province (No.145RJYA272), ASTIP, CAAS; NBCIS CARS38; Supporting Program (2013BAD12B03, 2013BAD12B05); Jiangsu Co-innovation Center programme for Prevention and Control of Important Animal Infectious Diseases and Zoonoses, State Key Laboratory of Veterinary Etiological Biology Project.

babalis, Linnaeus, 1758) in the Hubei province, South China. Vet. Parasitol. 186, 490-496. doi: 10.1016/j.vetpar.2011.11.021

Herwaldt, B. L., Cacciò, S., Gherlinzoni, F., Aspöck, H., Slemenda, S. B., Piccaluga, P., et al. (2003). Molecular characterization of a non-Babesia divergens organism causing zoonotic babesiosis in Europe. Emerg. Infect. Dis. 9, 942-948. doi: 10.3201/eid0908.020748

Inokuma, H., Yoshizaki, Y., Shimada, Y., Sakata, Y., Okuda, M., and Onishi, T. (2003). Epidemiological survey of Babesia species in Japan performed with specimens from ticks collected from dogs and detection of new Babesia DNA closely related to Babesia odocoilei and Babesia divergens DNA. J. Clin. Microbiol. 41, 3494-3498. doi: 10.1128/JCM.41.8.3494-3498.2003

Jiang, J.-F., Zheng, Y.-C., Jiang, R.-R., Li, H., Huo, Q.-B., Jiang, B.-G., et al. (2015). Epidemiological, clinical, and laboratory characteristics of 48 cases of "Babesia venatorum" infection in China: a descriptive study. Lancet Infect. Dis. 15, 196-203. doi: 10.1016/S1473-3099(14)71046-1

Karnath, C., Obiegala, A., Speck, S., Essbauer, S., Derschum, H., Scholz, H., et al. (2016). Detection of Babesia venatorum, Anaplasma phagocytophilum and Candidatus Neoehrlichia mikurensis in Ixodes persulcatus ticks from Mongolia. Ticks Tick Borne Dis. 7, 357-360. doi: 10.1016/j.ttbdis.2015.12.007

Kawabuchi, T., Tsuji, M., Sado, A., Matoba, Y., Asakawa, M., and Ishihara, C. (2005). Babesia microti-like parasites detected in feral raccoons (Procyon lotor) 
captured in Hokkaido, Japan. J. Vet. Med. Sci. 67, 825-827. doi: 10.1292/jvms. 67.825

Kik, M., Nijhof, A. M., Balk, J. A., and Jongejan, F. (2011). Babesia sp. EU1 infection in a forest reindeer, The Netherlands. Emerg. Infect. Dis. 17, 936-938. doi: 10.3201/eid1705.101834

Kim, C.-M., Blanco, L. B. C., Alhassan, A., Iseki, H., Yokoyama, N., Xuan, X., et al. (2008). Development of a rapid immunochromatographic test for simultaneous serodiagnosis of bovine babesioses caused by Babesia bovis and Babesia bigemina. Am. J. Trop. Med. Hyg. 78, 117-121.

Kimura, M. (1980). A simple method for estimating evolutionary rates of base substitutions through comparative studies of nucleotide sequences. J. Mol. Evol. 16, 111-120. doi: 10.1007/BF01731581

Lempereur, L., Beck, R., Fonseca, I., Marques, C., Duarte, A., Santos, M., et al. (2017). Guidelines for the detection of Babesia and Theileria parasites. Vector Borne Zoonotic Dis. 17, 51-65. doi: 10.1089/vbz.2016.1955

Liu, J., Guan, G., Liu, A., Li, Y., Yin, H., and Luo, J. (2014). A PCR method targeting internal transcribed spacers: the simultaneous detection of Babesia bigemina and Babesia bovis in cattle. Acta Parasitol. 59, 132-138. doi: 10.2478/s11686014-0222-6

Liu, J., Yang, J., Guan, G., Liu, A., Wang, B., Luo, J., et al. (2016). Molecular detection and identification of piroplasms in sika deer (Cervus nippon) from Jilin Province, China. Parasit. Vectors 9:156. doi: 10.1186/s13071-016-1435-3

Liu, J., Yin, H., Liu, G., Guan, G., Ma, M., Liu, A., et al. (2008). Discrimination of Babesia major and Babesia ovata based on ITS1-5.8S-ITS2 region sequences of rRNA gene. Parasitol. Res. 102, 709-713. doi: 10.1007/s00436-007-0818-y

Luo, J., Yin, H., Guan, G., Zhang, Q., and Lu, W. (2002). Description of a new Babesia sp. infective for cattle in China. Parasitol. Res. 88, S13-S15.

Malandrin, L., Jouglin, M., Sun, Y., Brisseau, N., and Chauvin, A. (2010). Redescription of Babesia capreoli (Enigk and Friedhoff, 1962) from roe deer (Capreolus capreolus): isolation, cultivation, host specificity, molecular characterisation and differentiation from Babesia divergens. Int. J. Parasitol. 40, 277-284. doi: 10.1016/j.ijpara.2009.08.008

Niu, Q., Liu, Z., Yu, P., Yang, J., Abdallah, M. O., Guan, G., et al. (2015). Genetic characterization and molecular survey of Babesia bovis, Babesia bigemina and Babesia ovata in cattle, dairy cattle and yaks in China. Parasit. Vectors 8:518. doi: 10.1186/s13071-015-1110-0

Qi, C., Zhou, D., Liu, J., Cheng, Z., Zhang, L., Wang, L., et al. (2011). Detection of Babesia divergens using molecular methods in anemic patients in Shandong
Province. China. Parasitol. Res. 109, 241-245. doi: 10.1007/s00436-0112382-8

Qin, S.-Y., Wang, J.-L., Ning, H.-R., Tan, Q.-D., Yin, M.-Y., Zhang, X.-X., et al. (2015). First report of Babesia bigemina infection in white yaks in China. Acta Trop. 145, 52-54. doi: 10.1016/j.actatropica.2015.02.005

Qin, S.-Y., Zhang, X.-X., Zhao, G.-H., Zhou, D.-H., Yin, M.-Y., Zhao, Q., et al. (2014). First report of Cryptosporidium spp. in white yaks in China. Parasit. Vectors 7:230. doi: 10.1186/1756-3305-7-230

Saravanan, B. C., Das, S., Siju, S. J., Tewari, A. K., Sankar, M., Kataktalware, M. A., et al. (2013). Babesia bigemina infection in yak (Poephagus grunniens L.): molecular detection and characterization. Vet. Parasitol. 194, 58-64. doi: 10.1016/j.vetpar.2012.12.024

Schnittger, L., Rodriguez, A. E., Florin-Christensen, M., and Morrison, D. A. (2012). Babesia: a world emerging. Infect. Genet. Evol. 12, 1788-1809. doi: 10.1016/j.meegid.2012.07.004

Sun, Y., Li, S.-G., Jiang, J.-F., Wang, X., Zhang, Y., Wang, H., et al. (2014). Babesia venatorum infection in child, China. Emerg. Infect. Dis. 20, 896-897. doi: 10.3201/eid2005.121034

Tamura, K., Stecher, G., Peterson, D., Filipski, A., and Kumar, S. (2013). MEGA6: molecular evolutionary genetics analysis version 6.0. Mol. Biol. Evol. 30, 2725-2729. doi: 10.1093/molbev/mst197

Yin, H., Lu, W., and Luo, J. (1997). Babesiosis in China. Trop. Anim. Health Prod. 29, 11S-15S. doi: 10.1007/bf02632908

Zanet, S., Trisciuoglio, A., Bottero, E., de Mera, I. G. F., Gortazar, C., Carpignano, M. G., et al. (2014). Piroplasmosis in wildlife: Babesia and Theileria affecting free-ranging ungulates and carnivores in the Italian Alps. Parasit. Vectors 7:70. doi: $10.1186 / 1756-3305-7-70$

Conflict of Interest Statement: The authors declare that the research was conducted in the absence of any commercial or financial relationships that could be construed as a potential conflict of interest.

Copyright (C) 2017 Liu, Guan, Li, Liu, Luo and Yin. This is an open-access article distributed under the terms of the Creative Commons Attribution License (CC BY). The use, distribution or reproduction in other forums is permitted, provided the original author(s) or licensor are credited and that the original publication in this journal is cited, in accordance with accepted academic practice. No use, distribution or reproduction is permitted which does not comply with these terms. 